\title{
Evaluation of common concussion tools used in a sport's setting
} BRADY ARMITAGE ${ }^{1}, *$ B. SUE GRAVES ${ }^{2}$

\author{
${ }^{1}$ Medical 1MF Division, DonJoy Orthopedics Global 2900 Lake Vista Drive, Lewisville, TX 75067 \\ UNITED STATES OF AMERICA \\ ${ }^{2}$ Department of Exercise Science and Health Promotion, College of Science, Florida Atlantic University \\ 777 Glades Road, Field House 11, Boca Raton, Florida 33431 \\ UNITED STATES OF AMERICA
}

\begin{abstract}
Sports medicine advancements are continuously evolving allowing professionals to utilize tools to provide for their athletes' care. These tools have allowed clinicians to better diagnose and determine the extent of an athlete's injury. Over the last 20 years, an emphasis has been placed on mild traumatic brain injuries (mTBI) and/or concussions. This focus on mTBI and concussions has led to an understanding of the mechanism of injury (MOI), development of grading/severity scales of injury, and diagnostic tools for properly assessing an athlete suffering from an injury to the brain. Clinicians understanding of concussion has excelled in recent years, but with advancement in technologies and diagnostic tools, all professionals need to understand the importance of incorporating tools into the diagnostic procedure. Thus, the purpose of this review is to evaluate common tools in practice, as well as newer tools, that could be utilized by sports medicine professionals.
\end{abstract}

Key-Words: - neurocognitive function, head injury, sport mTBI

Received: December 3, 2020. Revised: March 17, 2021. Accepted: March 25, 2021. Published: April 1, 2021.

\section{Introduction}

In today's society, sports have reached an all-time high of popularity and continue to grow. This growth has led to athletes of all ages participating in a variety of sports from youth to professional. With so many individuals participating in sport, athletes know a risk of injury exists with participation. In the past, focus has been heavily aimed at musculoskeletal and ligamentous injuries. A new focus of brain injuries, specifically the sport-related concussion, has become more prevalent and studied in recent years. Not to say that other types of injuries cannot be catastrophic, but individuals surrounding sport need to be more aware of head injuries due to less research surrounding the topic. According to Howell et al16, an estimated 1.6 - 3.8 million concussions occurred from sport annually and has increased from $23 \%$ to $57 \%$ from the year 2005 to 2012.

In addition, what makes the diagnosis of an mTBI more difficult is the variety of signs and symptoms $(\mathrm{S} / \mathrm{s})$ that could occur. Common signs and symptoms can include neurological deficits, visual, hearing, sensory and motor control deficits, nausea, and more depending on the site of injury ${ }^{16}$. Recently, mTBI's and concussions have become a main focal point in sports medicine research. Furthermore, current research surrounding the topic has focused heavily on incidence rates, significance levels, assessments and recovery from the injury ${ }^{4}$.
Though research has become more prevalent, it is still in its infancy compared to other topics in the field. As more information is discovered about concussions, the better sports medicine personnel will be able to properly diagnose a concussion. Thus, the purpose of this article is to identify the validity, sensitivity and specificity of common concussion tools used by clinicians.

\section{Defining and Recognition}

Individuals surrounding sport, whether that be coaches, athletes, athletic directors, parents and sports medicine personnel, need to be able to recognize common symptoms of a concussion to help better protect, but properly identify, someone at risk $^{16}$. These individuals will be around athletes more than a physician, who would give the clinical diagnosis, so the education of everyone will drastically help athletes and their protection. Numerous organizations like the National Collegiate Athletics Association (NCAA) and NATA (National Athletic Trainers' Association (NATA) recommend the education and baseline screening of athletes prior to participation ${ }^{13,23}$. Hootman ${ }^{15}$ reviewed injury rates in collegiate athletes to find that sport-related concussions accounted for five percent of all injuries across twenty sports ${ }^{15}$. 


\section{Education}

Perhaps one of the hardest factors in identifying a concussion is that the injury can be presented differently between individuals and based on where it occurred within the brain. The severity of concussions can range from mild to severe, so the importance to understand the types and grades at which concussions can be categorized. The two categories of concussions mentioned in the National Athletic Training Associations' position statement are focal and diffuse13. Focal or post-traumatic intracranial mass lesions to the brain can be presented through a wide variety of conditions including subdural hematoma, epidural hematomas, cerebral contusions, intracerebral hemorrhages and hematomas13. Injuries like cranial hematomoas and hemorrhages are less common, yet serious. These concussions that can occur within sport and the identification of these injuries can be a matter of saving a life. Typical conditions following a focal injury to the brain can include: loss of consciousness (LOC), cranial nerve deficits, mental deterioration and continued worsening symptoms 13 .

A diffuse brain injury can present a wide variety of injury with the most severe being injury to the brainstem, but more commonly presented is the cerebral concussion. A cerebral concussion, sometimes termed mild traumatic brain injury (mTBI), can best be categorized as a diffuse injury due to the mechanism of injury (MOI)13. Common diffuse injuries come from acceleration/deceleration and or rotational motions, sometimes including both13. This mechanism of injury causes a sudden change that can result in tissue damage. If tissue damage does occur, a variety of signs and symptoms can be presented depending on the severity and the location of the tissue damage. An injury to the frontal lobe may present differently than tissue damage to the occipital lobe, so consideration of location is important when evaluations are being completed. Therefore, signs and symptoms that can be presented from cerebral concussions may include: headache, dizziness, nausea, vomiting, balance problems, feeling "slowed down," fatigue, trouble sleeping, sensitivity to light or noise, LOC, blurred vision, difficulty with remembering, memory and concentration 13 .

\section{Grading Scale:}

As more information comes into light with concussions, varying grading scale or severity scales have been used to classify a concussion. However, not one scale can particularly be agreed upon, but similarities have been shown in three systems since the late 1990s3. Those scales coming from Cantu, the American Academy of Neurology, and the Colorado Medical Society ${ }^{3}$.see Table 1.

Table 1. Criteria of Concussion/mTBI Grading Scales*

\begin{tabular}{|c|c|c|c|}
\hline & Grade 1 (Mild) & Grade 2 (Moderate) & Grade 3 (Severe) \\
\hline Cantu & $\begin{array}{l}\text { No } \mathrm{LOC}^{1} \\
\text { PTA }^{2} / \mathrm{PCSS}^{3}<30 \mathrm{~min}\end{array}$ & $\begin{array}{l}\text { LOC }<1 \text { min or } \\
\text { PTA }>\min <24 \mathrm{hr} \\
\text { PCSS }>30 \mathrm{~min}, 7<\text { days }\end{array}$ & $\begin{array}{l}\text { LOC }>1 \mathrm{~min} \\
\text { PTA }>24 \mathrm{hr}\end{array}$ \\
\hline $\begin{array}{l}\text { Colorado } \\
\text { Medical } \\
\text { Society }\end{array}$ & $\begin{array}{l}\text { Confusion w/o amnesia } \\
\text { No LOC }\end{array}$ & $\begin{array}{l}\text { Confusion w amnesia } \\
\text { No LOC }\end{array}$ & Any LOC \\
\hline $\begin{array}{l}\text { American } \\
\text { Academy of } \\
\text { Neurology }\end{array}$ & $\begin{array}{l}\text { Transient confusion } \\
\text { No LOC } \\
\text { Symptoms or mental } \\
\text { status abnormalities } \\
\text { resolve within } 15 \text { min }\end{array}$ & $\begin{array}{l}\text { Transient confusion } \\
\text { No LOC } \\
\text { Symptoms or mental } \\
\text { status or abnormalities } \\
\text { last more than } 15 \text { min }\end{array}$ & Any LOC \\
\hline \multicolumn{4}{|c|}{$\begin{array}{l}{ }_{1}^{1} L O C, \text { Loss of consciousness } \\
{ }^{2} P T A \text {, posttraumatic amnesia } \\
{ }^{3} P C S S \text {, post-concussion symptom score }\end{array}$} \\
\hline
\end{tabular}

Cantu ${ }^{8}$ published the first scale to grade the severity of concussions: grade 1, mild, with no loss of consciousness; grade 2, moderate, with less than five minutes of unconsciousness or more than thirty minutes of post traumatic amnesia; grade 3 , severe, with five or more minutes of unconsciousness or 24 or more hours of post traumatic amnesia ${ }^{8}$.

The Colorado Medical Society used a grading scale similar to that of Cantu ${ }^{8}$ by measuring the amount of amnesia and LOC presented in an individual, which is seen in his proposal. The Colorado Medical Society grades are as follows: grade 1, confusion without amnesia or LOC; grade 2 , confusion with amnesia without LOC and grade 3 , being any $\mathrm{LOC}^{3}$. To expand upon this scale, The American Academy of Neurology (AAN) added further criteria to their scale. The AAN added a scale to measure how long symptoms or mental status abnormalities were present. The difference between grade 1 and 2 was the length of these abnormalities being less than 15 minutes is considered grade 1 , while over 15 minutes was considered grade 2 concussion $^{3,13}$. With adherence to the Colorado Medical Society Scale, any LOC was considered a grade 3 concussion. In today's NATA position statement ${ }^{13}$, the recommendation of these three scales is the American Academy of Neurology.

\section{Evaluation Tools:}

Sport Concussion Assessment Tool (SCAT). The SCAT, now on its $5^{\text {th }}$ edition, is a memory and symptom assessment tool that can be used to perform an in depth assessment for an individual 
suspected of a concussion ${ }^{2}$. If done properly, the SCAT assessment should take no less than 10 minutes. Basis of the SCAT is the Standardized Assessment of Concussion (SAC), Post-Concussion Symptom Scale (PCSS), which includes a 22 selfreport scale rating from 0 (none) to 6 (severe), as well as a modified Balance Error Scoring System (BESS), in addition to numerous other sections within the SCAT to thoroughly evaluate a variety of brain functions ${ }^{21}$.Preseason SCAT baseline testing can be useful for interpreting post-injury test scores also. The diagnosis of a concussion is a clinical judgment, made by a medical professional.

Balance Error Scoring System (BESS). The BESS Test is a postural stability measurement designed to evaluate concussed and healthy athletes in a quick manner. Figure 1 shows that the athlete is asked to perform numerous stances (double leg, single leg, tandem stance) both eyes open or closed for twenty seconds as errors are counted throughout the exam $^{5,7,24}$.

The BESS Test is a vital component to incorporate into concussion examinations, because numerous studies have shown that balance can be altered for three to ten days in most cases, typically resolving in the first three to five days, but in extreme cases, 28-37 days can be the time period ${ }^{1,10,16,21,24,25}$. Ozinga et al. (2018) have shown age as an impactful factor on both baseline and concussed scores. This impact is thought to occur at younger ages, because sensory system and automatic motor process have not fully developed ${ }^{24}$.

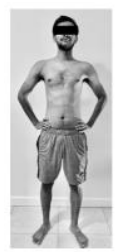

(a)

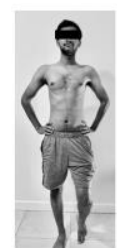

(b)

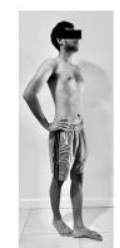

(c)

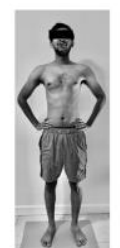

(d)

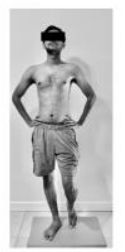

(e)

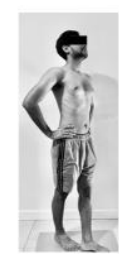

(f)
Figure 1. Balance Error Scoring System (BESS). Top row, firm surface condition Bottom row, soft surface condition, Left column, parallel stance, Middle column, single-leg stance, Right column, Tandem stance

Immediate Post-Concussion Assessment and Cognitive Testing (ImPACT Test). ImPACT is a commonly used baseline tool requiring 25-30 minutes as shown in figure 2. This tool provides a 22 question post-concussion symptoms score, as well as six neurocognitive subjects that include attention span, working memory, sustained and selective attention time, response variability, nonverbal problem solving, and reaction time during the test ${ }^{7,9}$.

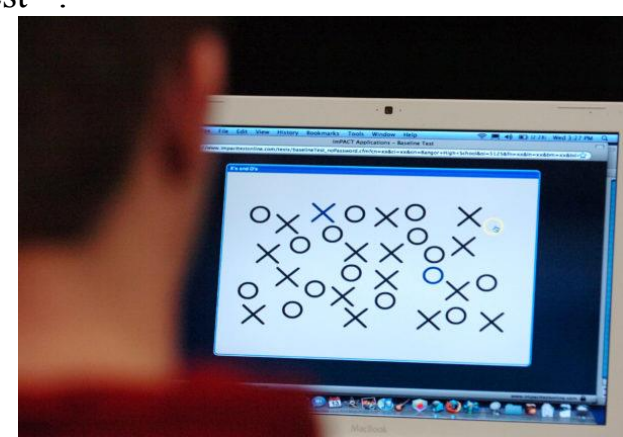

Figure 2. ImPACT Test Assessment

(https://www.juniorhockey.com/news/news_detail.p $h p$ ?news_id=79867)

A composite score from various domains evaluates verbal memory, visual memory, processing speed and reaction time ${ }^{9}$. Similar computerized tools like the ImPACT Test are available, such as the Automated Neuropsychological Assessment Metrics (ANAM), CogSport, and Concussion Resolution Index. Each supporting computerized test incorporates similar examinations of memory and reaction time ${ }^{13}$.

Vestibular Ocular Motor Screen (VOMS). The VOMS testis five- to ten-minute visual and ocular function screening through which an individual is required to complete an eye-tracking screen, specifically measuring visual acuity, smooth pursuits, saccades, and ability to focus on stationary objects $^{5,19,21}$. This specific assessment puts additional strain on the vestibular complex, providing information regarding head movements, visual and balance control ${ }^{21}$. Due to the vestibular system providing feedback from balance and visual acuities, thus, the VOMS test needs to be divided into subsections because of the uniqueness of each area.

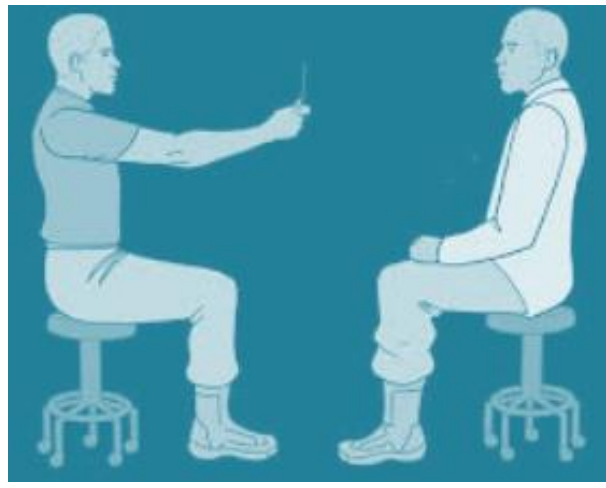

Figure 3. Face to Face Vestibular Ocular Motor Screen

Face to Face Vestibular Ocular Motor Screen shown in Figure 3. Those two subsections are the vestibulo-ocular system, which is responsible for maintaining visual fields, and the vestibule-spinal 
system, being responsible for controlling postural stability $^{21}$. The VOMS test, unlike the BESS and SOT test, adds a dynamic component to vestibular and vestibulo-ocular motor systems ${ }^{21}$.

King-Devick Test. The King-Devick Test consists of a series of four cards. The first card is used as a practice or introduction card to familiarize the individual with the test. Each of the following cards can be termed as exam cards, where the clinician will take a time for the reading of each $\operatorname{card}^{7,19}$. The athlete is asked to read a series of numbers with varying spacing between cards as quickly and accurately as possible ${ }^{7,19,27}$. Additionally, errors are counted if numbers are skipped or incorrectly read. Upon completion, the time and total numbers of errors are corrected ${ }^{7}$. Although the King-Devick Test provides an object measurement for sport related concussions, it is limited to only to testing oculomotor impairment excluding any vestibular components ${ }^{19}$.

Trazer. The Trazer is a versatile piece of equipment (television with an operating and analysis system attached)that can be used to evaluate sensory, cognitive and neuromuscular system through evaluation of balance, posture, agility and reaction time as shown in Figure 4.

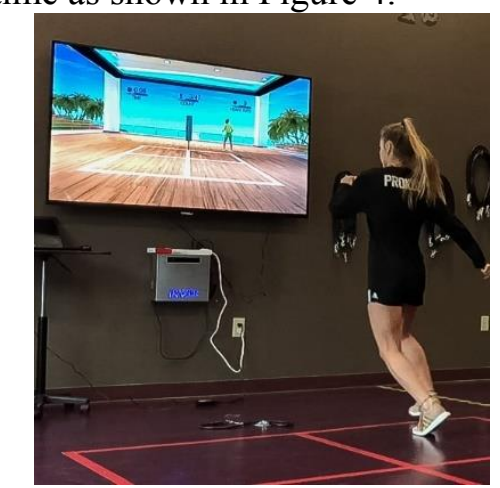

Figure 4. Testing point of TRAZER

Also, the Trazer analyzes movements of the lower extremity, more specifically the knee and ankle joints. Though, not much research has been published on the TRAZER, the components in which the TRAZER does focus on related to concussion are visual, vestibular, cognitive, neuromuscular/musculoskeletal and cardiorespiratory systems ${ }^{32}$. Furthermore, two collegiate football programs (University of Alabama \& Troy University) have been shown to use the TRAZER in their baseline assessment ${ }^{14,32}$.

EquiTest (Sensory Organization Test, SOT). Figure 5 represents a visual of the SOT has recently become a versatile tool in concussion management, as it can be utilized to measure vestibular, somatosensory, and neurocognitive function on an individual ${ }^{22,26,30,33}$.
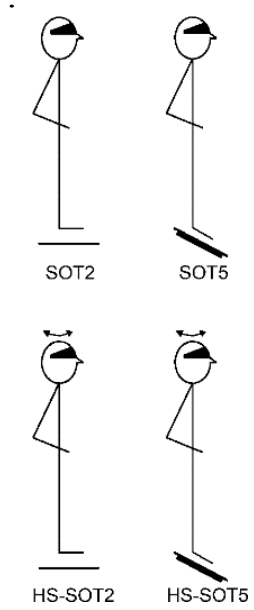

Figure 5 EquiTest (Sensory Organization Test, SOT) Position

The measurements are derived from the utilization of a force plate, reference point, anterior and posterior tilting of the force plate, and the surrounding area during the test disrupts somatosensory or visual inputs ${ }^{22}$. The SOT allows clinicians to objectively measure abnormalities in the sensory systems assisting with posture stability $^{22,33}$.

Virtual Reality (VR) Technology. Virtual reality tools allow for a level of engagement and sense of presence by simulating daily activities while providing a measureable stimulus ${ }^{34}$ as shown in Figure 6. VR technology has allowed clinicians to integrate vestibular, visual, and somatosensory information, which have all are potentially present in a concussed athlete ${ }^{30}$.

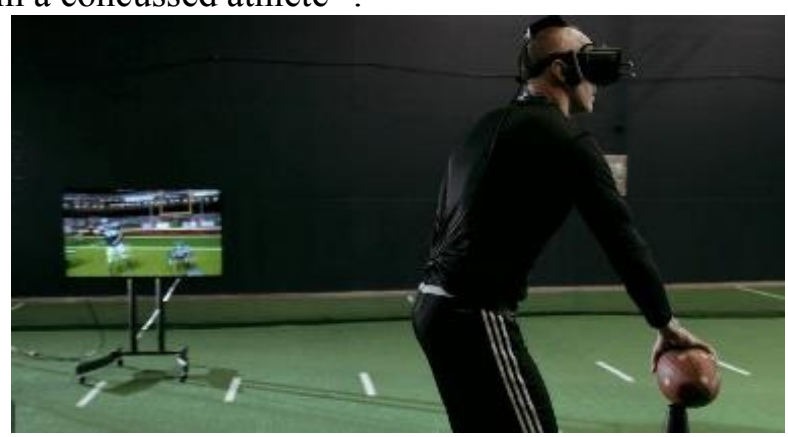

Figure 6 Virtual Reality (VR) Technology position Current research focus has been placed on components, such as visual, somatosensory and balance and anticipatory rates ${ }^{20,30}$. Numerous tools have been developed to measure an athlete suspected of a concussion or mTBI by evaluating eye movement that identifies abnormalities in the athlete's baseline measure ${ }^{20}$. VR tools can be compared to the VOMS, but are also thought to measure predictive timing ${ }^{20}$. By providing a measurement for predictive timing in athletes, 
clinicians and sports medicine team can determine if an athletes' performance is deemed unsafe to return to competition.

\section{Reliability, Sensitivity, and Specificity of These Tools :}

Sport Concussion Assessment Tool (SCAT) (Broken down into subsections, SAC, PCSS, mBESS). Sport medicine professionals have utilized the SCAT since 2004 when it was introduced at the International Conference on Concussion in Sport in Prague $^{2}$. The SCAT is now on the 5 th version and still widely used today. Putukian et al. (2015) looked into the specificity and sensitivity of detecting a concussion ${ }^{27}$. Upon review, concussed individuals score dropped 3.5 points compared to baseline scores $^{27}$. Furthermore, a $96 \%$ sensitivity and $81 \%$ specificity in detecting individuals suspected of concussion was determined ${ }^{27}$. Though the SCAT 2 has been shown to be a valid tool for health care professionals, an updated review of the SCAT 5 should be conducted to test reliability, since more has been added to the evaluation. The SCAT has since added a cervical spine and neurological screening, plus has given the clinician more questions on observational signs.

Balance Error Scoring System (BESS). The Balance Error Scoring System has been shown to have a low sensitivity, with high specificity, when diagnosing sport-related concussions. Buckley et al.(2016) observed that the sensitivity and specificity of the modified BESS were $71.4 \%$ and $65.7 \%$, respectively, for collegiate athletes with acute sport-related concussions. ${ }^{6}$ However, when post-injury results were compared with baseline values, $60 \%$ of participants were misclassified at some point during the testing protocol (acute or recovery) ${ }^{1}$. Furthermore, Baracks et al.(2018) found the BESS test presented differences in balance analyzes ${ }^{1}$. To support Barrack's claimed sensitivity and specificity, Caccese ${ }^{7}$ found a low sensitivity (.34) and high specificity (.91-.97) for the BESS test? ${ }^{7}$.

Immediate Post-Concussion Assessment and Cognitive Testing (ImPACT). Elbin et al. (2019) found no significant improvements on the ImPACT test when retaking the test on the same day in healthy individuals. ${ }^{9}$ Therefore, the ImPACT test can be a suitable tool within a clinician's diagnosis protocol due to the unlikely hood of improvements, even with familiarity of the examination. Only slight improvements were found on visual motor speed, while all other measurements remained stable $^{28}$. Furthermore, the ImPACT Test is shown to have a sensitivity of $81.9 \%$ and a specificity of
$89.4 \%$ when combining symptom scores and the ImPACT test ${ }^{7,28}$. Also, ImPACT data revealed an $85 \%$ diagnosis rate of concussion ${ }^{29}$.

Vestibular/Ocular-Motor

(VOMS). Mucha et al.(2014) obseening individuals, who suffered an mTBI, had a total mean symptom score on the VOMS significantly lower than that of non-concussed individual $(<.001)^{20}$. Also, a positive correlation between each VOMS domain to the Post Concussion Symptom scale (.28-.65) was seen ${ }^{19,20}$. However, further research on validity is needed due to the wide scope of the examination to specifically identify areas of deficit. Thoughadditional research is necessary, this test could be used for rehabilitation protocols of sport-related concussions due to the wide scope of tests given and ease of administration.

King-Devick Test. Previous research has validated that the King-Devick test can recognize a sport related concussion (SRC) when an athlete's Post-Concussion Symptom Scale (PCSS) does not reflect possible concussion ${ }^{12,18,19}$. Furthermore, the King-Devick Test has shown similar deficits in scores on visual motor speed and reaction time to thatof the SCAT and ImPACT Test ${ }^{18,19}$. Additionally, concussed football athletes were measured at baseline and post concussion to show an average $46.9 \mathrm{sec}$ to 37.0 seconds at baseline to complete the exam ${ }^{12}$. Thus, the King-Devick can be a reliable tool, even showing a high sensitivity (1.00) and specificity (.94) in test scores following concussion? ${ }^{7}$. However, conflicting evidence has shown the King-Devick test is predisposed to practice effects, indicating a performance improvement over time ${ }^{19,27}$. Though, conflicting evidence has been presented, Putukian et al. (2015) stated the importance of a baseline testing to compare scores when a concussion is suspected to limit this adaption ${ }^{27}$.

TRAZER. Current published peer-reviewed research does not exist to our knowledge on the TRAZER's effectiveness as a tool to manage concussions. Though the TRAZER's effectiveness has not been studied thoroughly, information on the various domains of focus when using their concussion protocol are prevalent ${ }^{32}$. The TRAZER does incorporate numerous domains found on other concussion tools, such as neurocognitive functions, metabolic function, and dynamic movement analysis. Thus, the TRAZER could be a versatile tool in a clinicians' diagnosis procedure, if a thorough understanding of the tool and domains of focus are known when adding this tool to a concussion return to play protocols. 
EquiTest (SOT). Though the popularity of the SOT has become more prevalent in recent years, evidence is limited and needs to be interpreted rationally. Numerous studies have shown the SOT to be a viable tool for objectively measuring individuals' balance and postural control ${ }^{11,22,33}$. In addition, FordSmith and colleagues found deficits in postconcussion symptom scores compared to baseline scores, specifically in total score and vestibular impairment ${ }^{11,17}$. Individuals, also, following vestibulo-oculomotor training, saw significant improvements in symptoms upon follow up testing ${ }^{11}$. On the contrary, Wisely et al, found the SOT to be an unreliable tool when managing an athlete suspected of concussion ${ }^{31}$. Their findings indicated a large variety in scores, but the specific population tested was significantly older (average age $>60$ ) than the previous studies mentioned above, thus, adding thought to the population when administering tests, when measuring possible concussions, is important.

Virtual Reality (VR) Technology. Virtual reality tools have started to become a relevant tool used in concussion management ${ }^{21,30,34}$. The advancement in technology has allowed VR to bring components of clinic tools to one device for quick evaluation with objective measurements ${ }^{30}$. Research is still in the infancy of using these tools for concussion protocols, but Teel et al. (2016) investigated the sensitivity and specificity of balance in concussed athletes $^{30}$. They found a sensitivity of $87.7 \%$ and a specificity of $85.7 \%$ when measuring an athlete's balance post-concussion. Additionally stated, a $65.7 \%$ positive predictive value was found, plus a $97.7 \%$ negative predictive value when looking at the balance of concussed athletes ${ }^{30}$. Mucha and others investigated eye tracking and predicative measures using vertical, horizontal and circular tracks ${ }^{21}$. These same researchers did not see a significant difference in groups, most likely due to the population being healthy adults, although a correlation of horizontal and vertical tracking was seen, if either performed at a low score ${ }^{21}$. Furthermore, research is just in the infancy of using VR technology in brain injuries, but provides clinicians and the sports medicine a direction on where research needs to grow.

\section{CONCLUSION:}

Sports medicine professionals are able to utilize a wide variety of tools, covering numerous domains that can provide optimal care for their athletes. The tools mentioned have allowed for these clinicians to better diagnose and determine the extent of an athlete's injury. An emphasis will still be placed on the continuation of concussion research. That focus should be directed toward the mechanism of injury (MOI), severity of injury, or current and new diagnostic tools effectiveness, sensitivity, and specificity in determining if an athlete has suffered a concussion or mTBI. The knowledge of a clinicians' understanding of concussion has excelled in the last 20 years and will continue to grow with the advancement of research and diagnostic tools.

\section{Consent:}

Informed consent was obtained from the patient to publish case details, test results, and images.

Competing interests:

No competing interests to disclose.

\section{References:}

[1]. Baracks J, Casa D, Covassin T, Sacko R, Scarneo S, Schnyer D, Yeargin S, Neville C. Acute sport-related concussion screening for collegiate athletes using an instrumented balance assessment. J Athl Train. 2018;53(6):597-605.

[2]. Begasse de Dhaem O, Barr W, Balcer L, Galetta S, Minen M. Post-traumatic headache: the use of the sport concussion assessment tool (SCAT-3) as a predictor of post-concussion recovery. J Headache Pain. 2017;18(1).

[3]. Bodin D, Yeates K, Klamar K. Definition and classification of concussion. In Pediatric and adolescent concussion. In Apps JN, Walter KD, eds. Pediatric And Adolescent Concussion. 1st ed. New York, NY: Springer; 2012:9-19.

[4]. Broglio S, Collins M, Williams R, Mucha A, Kontos A. Current and Emerging Rehabilitation for Concussion. Clin Sports Med. 2015;34(2):213-231.

[5]. Broglio S, Katz B, Zhao S, McCrea M, McAllister T. Correction to: Test-Retest Reliability and Interpretation of Common Concussion Assessment Tools: Findings from the NCAA-dod CARE Consortium. Sports Med. 2018:48(7):1761-1761.

[6]. Buckley T, Munkasy B, Clouse B. Sensitivity and Specificity of the Modified Balance Error Scoring System in Concussed Collegiate Student Athletes. Clin J Sport Med. 2018;28(2):174-176.

[7]. Caccese J, Best C, Lamond L et al. Effects of Repetitive Head Impacts on a Concussion Assessment Battery. Med Science Sports Ex. 2019;1. 
[8]. Cantu R. Guidelines for Return to Contact Sports After a Cerebral Concussion. Phys Sportsmed. 1986;14(10):75-83.

[9]. Elbin R, Fazio-Sumrok V, Anderson M, D'Amico N, Said A, Grossel A, Schatz P, Lipinski D, Womble M. Evaluating the suitability of the Immediate

[10]. Post-Concussion Assessment and Cognitive Testing (ImPACT) computerized neurocognitive battery for short-term, serial assessment of neurocognitive functioning. $\mathrm{J}$ Clin Neuros. 2019;62:138-141.

[11]. Fait P, Swaine B, Cantin J, Leblond J, McFadyen B. Altered Integrated Locomotor and Cognitive Function in Elite Athletes 30 Days Postconcussion. J Head Trauma Rehab. 2013;28(4):293-301.

[12]. Ford-Smith C, Wyman J, Elswick R, Fernandez T, Newton R. Test-retest reliability of the sensory organization test in noninstitutionalized older adults. Arch Phys Med Rehab. 1995;76(1):77-81.

[13]. Galetta K, Brandes L, Maki K, Dziemianowicz M, Laudano E, Allen M, Lawler K, Sennett B, Wiebe D, Devick S, Messner L, Galetta S, Balcer L. The KingDevick test and sports-related concussion: Study of a rapid visual screening tool in a collegiate cohort. J Neurol Sciences. 2011;309(1-2):34-39.

[14]. Guskiewicz K, Bruce S, Cantu R et al. Recommendations on Management of Sport-related Concussion: Summary of the National Athletic Trainers' Association Position Statement. Neurosurgery. 2004;55(4):891-896.

[15]. Fitness-gaming Team website, TRAZER HRA Featured at the University of Alabama Football Program. https://fitness-gaming.com/news/healthand-rehab/trazer-hra.html. Accessed January 29, 2020.

[16]. Hootman J, Dick R, Agel J. Epidemiology of collegiate injuries for 15 sports: summary and recommendations for injury prevention initiatives. J Athl Train. 2007;42.

[17]. Howell D, Osternig L, Chou L. Dual-Task Effect on Gait Balance Control in Adolescents With Concussion. Arch Phys Med Rehabil. 2013;94(8):1513-1520.

[18]. Karin M, Nina F, Cornelia M et al. Integration of computerized dynamic posturography (equitest) in the rehabilitation process after concussion. $\mathrm{Br} \mathrm{J}$ Sports Med. 2019;51(11):A6.2-A6.

[19]. King D, Brughelli M, Hume P, Gissane C. Concussion in amateur rugby union identified with the used of rapid visual screening tool. J Neurol Sci. 2013;326(1-2):59-63.

[20]. Kontos A, Deitrick J, Collins M, Mucha A. Review of Vestibular and Oculomotor Screening and Concussion Rehabilitation. J Athl Train. 2017;52(3):256-261.

[21]. Maruta J, Heaton K, Kryskow E, Maule A, Ghajar J. Dynamic visuomotor synchronization: Quantification of predictive timing. Behav Res Methods. 2012;45(1):289-300.

[22]. Mucha A, Collins M, Elbin R et al. A Brief Vestibular/Ocular Motor Screening (VOMS) Assessment to Evaluate Concussions. Am J Sports Med. 2014;42(10):2479-2486.

[23]. Murray N, Salvatore A, Powell D, Reed-Jones R. Reliability and Validity Evidence of Multiple Balance Assessments in Athletes With a Concussion. J Athl Train. 2014;49(4):540-549.

[24]. National Collegiate Athletic Association Sport Institute Interassociation Consensus: Diagnosis and Management Of Sport-Related Concussion Best Practices. https://www.ncaa.org/sites/default/files/SSI ConcussionBestPractices_20170616.pdf.

$\bar{P}$ ublished February 2019. Accessed January 29, 2020.

[25]. Ozinga S, Linder S, Koop $\mathrm{M}$ et al. Normative Performance on the Balance Error Scoring System by Youth, High School, and Collegiate Athletes. J Athl Train. 2018;53(7):636-645.

[26]. Parker T, Osternig L, van Donkelaar P, Chou L. Recovery of cognitive and dynamic motor function following concussion. $\mathrm{Br} \mathrm{J}$ Sports Med. 2007;41(12):868-873.

[27]. Peterka R, Murchison C, Parrington L, Fino P, King L. Implementation of a Central Sensorimotor Integration Test for Characterization of Human Balance Control During Stance. Front Neurol. 2018;9.

[28]. Putukian M, Echemendia R, Dettwiler-Danspeckgruber A et al. Prospective Clinical Assessment Using Sideline Concussion Assessment Tool-2 Testing in the Evaluation of Sport-Related 
Concussion in College Athletes. Clin J Sport Med. 2015;25(1):36-42.

[29]. Schatz P, Ferris C. One-Month Test-Retest Reliability of the ImPACT Test Battery. Archives of Clinical Neuropsychology. 2013;28(5):499-504.

[30]. Schatz P, Pardini J, Lovell M, Collins M, Podell K. Sensitivity and specificity of the ImPACT Test Battery for concussion in athletes. Arch Clin Neurop. 2006;21(1):91-99.

[31]. Teel E, Gay M, Arnett P, Slobounov S. Differential Sensitivity Between a Virtual Reality Balance Module and Clinically Used Concussion Balance Modalities. Clin J Sport Med. 2016;26(2):162-166.

[32]. Wrisley D, Stephens M, Mosley S, Wojnowski A, Duffy J, Burkard R. Learning Effects of Repetitive Administrations of the Sensory Organization Test in Healthy Young Adults. Arch Phys Med Rehabil. 2007;88(8):10491054.

[33]. Von Thorn LA. Troy University First Collegiate Football Program To Test New Movement Analysis System. TRAZER. http://www.trazer.com/troyuniversity-first-collegiate-football-programtest-new-movement-analysis-system/. Published November 10, 2016. Accessed January 29, 2020.

[34]. Yuntao Z, Kondo I, Mukaino M et al. Reliability and validity of a forceinstrumented treadmill for evaluating balance: A preliminary study of feasibility in healthy young adults. Hong Kong Physiother J. 2017;36:49-56.

[35]. Zanier E, Zoerle T, Di Lernia D, Riva G. Virtual Reality for Traumatic Brain Injury. Front Neurol. 2018;9.

\section{Creative Commons Attribution License 4.0 (Attribution 4.0 International , CC BY 4.0)}

This article is published under the terms of the Creative Commons Attribution License 4.0 https://creativecommons.org/licenses/by/4.0/deed.en US 